\title{
Showing Relationship in Between a Same Reaction in both Liquid and Gas Phase by Monatomic Oxidation Hypothesis
}

\author{
Cemil Koyunoğlu ${ }^{1,2} *$ \\ ${ }^{1}$ Energy Institute, Istanbul Technical University, Sarlyer, Istanbul, 34469, Turkey \\ ${ }^{2}$ EMS Energy Institute, The Pennsylvania State University, University Park, State College, 16802, USA \\ *Corresponding Author: Cemil Koyunoğlu, Energy Institute, Istanbul Technical University, Sarlyer, \\ Istanbul, 34469, Turkey
}

\begin{abstract}
The new hypothesis based on "heat of a reaction which occurs in both the gas system, and the liquid system should be equal". In the early 19th century, William Henry stated that the concentration of the gas in the "gas system" would be directly proportional to its concentration in the "liquid system" at the constant temperature. However, Henry's law coefficient mainly varies with the temperature of the scheme. Also, Gustav Kirchoff described the "heat capacity", which is equal to change in enthalpy-introduced by Josiah Willard Gibbs in 1870's, then first called "enthalpy" in J. P. Dalton's scientific paper, in 1909-divided by the change in temperature, at constant pressure, in 1858's. This inference is based on the mathematical expression of the single atomic hypothesis. This hypothesis is often derived from a reaction that produces the same product output in two different systems to explain the ongoing events with each other. For example, in order for a coal particle to burn well, it is also important to cause the coal particle to ignite earlier during combustion, which is also an oxidation reaction, due to the oxidation reaction in water.
\end{abstract}

Keywords: Monatomic oxidation; Boltzmann entropy; Boltzmann probability; Gibbs free energy

\section{INTRODUCTION}

The oxidation reaction of the coal with an oxidizing agent in the liquid is directly related to the change in the energy resulting from a change in the surface area of the coal. There are two methods in which entropy can be changed. The first is that the number of atoms on the surface of the coal changes without the presence of stress applied to the surface. The explanation of this can be explained as follows. If the number of atoms on the surface of coal increases, this occurs as a result of the breakage of atomic bonds on the surface. The surface tension of coal, called a, increases or decreases depending on the energy of the broken bonds (Gibbs defined this as a new surface). Another second entropy change is that a deformation of the coal surface and the number of surface atoms remain the same. The second way of the change of S, also called surface tension, as a result of the presence of elastic forces due to this change in surface area, consists of the deformation of the surface without making any change in the number of surface atoms. The energy due to the change in the surface area is determined by a certain amount of elastic forces and therefore surface tension occurs. The deformation of the surface occurs spontaneously if the sample has a finite average curvature and has the same characteristics as the surface flexing.

\section{Methodology}

The emergence of this hypothesis has been developed to describe the association of the hydrogen atom on the surface of the coal with the oxygen atom in both liquid and gas medium. For this, the hypothesis was first defined according to entropy formulation of boltzman, then the probability of two atoms to meet according to boltzman probability law, and then the requirements for application of the formulation developed according to the gibbs principle are given below respectively.

\section{Derivation of the hypothesis}

the boltzmann entropy: Boltzmann's accomplishment has bestowed with an engraving of the formula (1) on his tombstone [1]. The symbol "S" signifies the entropy. The symbol W represents the German word Wahrscheinlichkeit, which suggests ‘probability' [2]. Oddly enough, Boltzmann never composed 
this equation, although it does precisely reflect his ideas [2]. The formula was composed initially of this type by the German physicist Max Planck (1858-1947) in 1900 [2]. The constant k refers to as the Boltzmann constant [2-4].

$\mathrm{S}=\mathrm{k} . \log \mathrm{W}$

the boltzmann probability: The Boltzmann probability distribution specifies that for a system that remains in thermal balance at absolute temperature level " $T$ ", the discovering the system probability $\mathrm{P}(\mathrm{E})$ in a state that has energy $(\mathrm{E})$ is offered by:

$P(E)=\frac{1}{Z} e^{-\frac{E}{k T}}$

Here E remains in joules $(\mathrm{J})$, T stays in kelvins $(\mathrm{K})$, and $\mathrm{k}$ is the Boltzmann constant with value $1.3806503 \times 10^{-23}$ joule per kelvin $(\mathrm{J} / \mathrm{K})[5]$. The denominator, $\mathrm{Z}$, called the partition function, is figured out so about make the amount of probabilities of all states of the system (i.e., the probability that the system remains in some state) equivalent to one [5-7].

the gibbs free energy: The change in Gibbs free energy in between two various chemical substances (compounds or conformations) that identifies the position of the balance between these states [8-11]. We signify the Gibbs free energy change for any procedure as $\Delta G^{o}$. Here, the $\Delta G^{o}$ of change is the finest idea of as the distinction instability of two various compositions of an ensemble of particles at constant states and constant pressure [8]. A more exact meaning is that Gibbs free energy is similar to a driving force (or a prospective power) for a spontaneous change in composition [8].

Formula (3) provides the relationship between the stability constant, $K_{\mathrm{eq}}$, and the free energy change for any chemical process, $\Delta G^{o}$ (the "o" represents we are thinking about basic states) Equation (4) provides the ratio of types $\mathrm{A}$ and $\mathrm{B}$, where $\mathrm{B}$ has less internal energy [8].

$\ln \mathrm{K}_{\mathrm{eq}}=\frac{-\Delta G^{o}}{R T}$

$\mathrm{K}_{\mathrm{eq}}=\frac{[B]}{[A]}$

Remember that the square brackets in the balance relationship provided (4) designate concentration, determined in $\mathrm{mol} / \mathrm{L}[8]$.

When the Gibbs totally free energy of B is lower than A, the change of A to B is exergonic. If we begin with excess $A$, an inevitable shift in the composition of the solution will happen to produce $B[8]$. Conversely, when the free energy of B is greater than A, the reaction is endergonic [8]. Use these terms when analyzing a Gibbs free energy diagram. Various terms ("exothermic" and "endothermic") use when the power axis is enthalpy [8]. Keep in mind that when we state "an inevitable change will happen" we are indicating nothing about the length of time will consider for that change to take place [8]. Thermodynamics just informs us the instructions where the modification will happen; we require kinetics to offer a timescale for the improvement [8].

The free energy has two elements, the enthalpy $\left(\Delta H^{o}\right)$ and the entropy $\left(\Delta S^{o}\right)$, related by the GibbsHelmholtz equation Formula (5) [8]. Enthalpy determines in $\mathrm{kcal} / \mathrm{mol}$, and entropy in entropy units (eu), which are comparable to cal/mol.K [8]. Therefore, for entropy to be an energy value, it should be increased by the temperature level [8]. This level has an essential effect. Modifications in temperature level impact the free energy between $\mathrm{A}$ and $\mathrm{B}$, and for that reason the equilibrium constant [8]. Integrating formula (3) and equation (5), we get Equation (5), revealing how a balanced constant is affected by temperature level [8, 12-14].

$\Delta G^{o}=\Delta H^{o}-T \Delta S^{o}$

$\ln \mathrm{K}_{\mathrm{eq}}=-\frac{\Delta H^{o}}{R T}+\frac{\Delta S^{o}}{R}$

\section{The Formulation of monatomic oxidation hypothesis}

The reaction between hydrogen and oxygen in elementary form is

$\mathrm{H}+\mathrm{O}$

$\mathrm{HO}$

for the atmospheric conditions the state is in the gas phase (State 1), for the oceans and seas, the state is liquid (State 2), so we have two states (Figure 1(a,b) and 2(a,b)). 

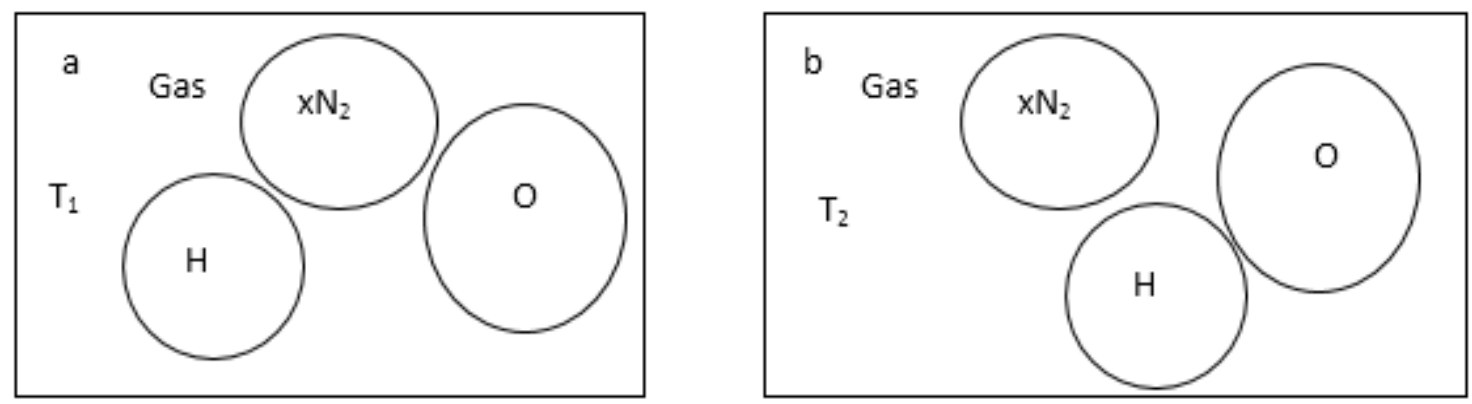

Figure1(a,b). Boltzmann probability reaction of an $H$ atom and an $O$ atom surrounded with $(x)$ number of a Nitrogen molecule $\left(\mathrm{N}_{2}\right)$ in an air atmosphere (gas stage)

a) $\mathrm{H}$ and $\mathrm{O}$ reaction in temperature, $\left.T_{1}, b\right) \mathrm{OH}$ production in temperature, $T_{2}$.
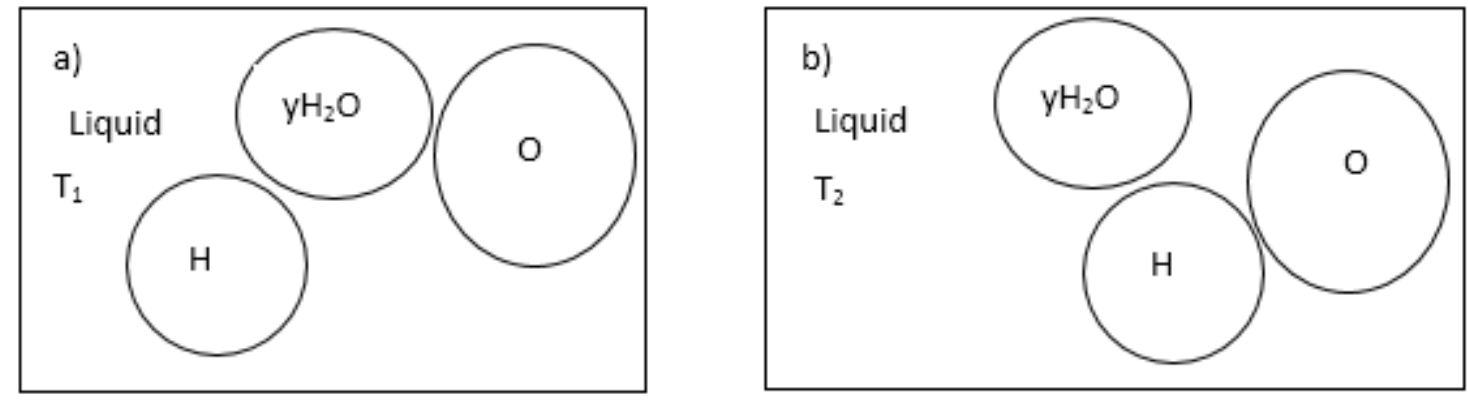

Figure2(a,b). Boltzmann probability reaction of an $H$ atom and an $O$ atom surrounded with a (y) number of a water molecule $\left(\mathrm{H}_{2} \mathrm{O}\right)$ in ocean and sea region (liquid stage). (a) $\mathrm{H}$ atom and $\mathrm{O}$ atom reaction in temperature, $T_{1}$, (b) OH production in temperature, $T_{2}$.

For State 1;

The natural logarithm of equation (2) is (State $1, Z=1$ ),

$\ln \left(\mathrm{P}_{1}(\mathrm{E})\right)=-\frac{E_{1}}{k T_{1}}$

if we extract $T_{1}$ from equation (6),

$\mathrm{T}_{1}=-\frac{E_{1}}{k \ln \left(\Delta \mathrm{P}_{1}(\mathrm{E})\right)}$

For State 1 if we extract $T_{1}$ from equation (3),

$\mathrm{T}_{1}=\frac{\Delta H_{1}{ }^{o}}{\Delta S_{1}{ }^{o}-R \ln K_{\mathrm{eq}(1)}}$

and $\Delta H_{1}{ }^{o}$ is equal

$\Delta H_{1}{ }^{o}=T_{1} \cdot\left(\Delta S_{1}{ }^{o}-R \ln \mathrm{K}_{\mathrm{eq}(1)}\right)$

calculation $\Delta S^{o}$ for the first state is from equation (6),

$\Delta S^{o}=\mathrm{k} \log \left(\frac{W_{2}}{W_{1}}\right)=\mathrm{k} \ln \left(\frac{W_{2}}{W_{1}}-10\right)$

the equation (11) becomes

$\Delta H_{1}{ }^{o}=T_{1} \cdot \ln \left(\frac{\left(\frac{W_{2}}{W_{1}}-10\right)^{k}}{\left(K_{e q(1)}\right)^{R}}\right)$

if we put equation (9) into equation (13),

$\Delta H_{1}{ }^{o}=-E_{1} \cdot \ln \left[\frac{\left(\frac{W_{2}}{W_{1}}-10\right)^{k}}{\left(K_{e q(1)}\right)^{R}}-\Delta P_{1}(E)^{-k}\right]$ 
For the State 2

$\Delta H_{2}{ }^{o}=-E_{2} \cdot \ln \left[\frac{\left(\frac{W_{2}}{W_{1}}-10\right)^{k}}{\left(K_{e q(2)}\right)^{R}}-\Delta P_{2}(E)^{-k}\right]$

Regulations of monatomic oxidation hypothesis

To equal heat of reaction of both (14) and (15)

- For the logarithm rules,

$\mathrm{W}_{2} / \mathrm{W}_{1}$ must be equal and higher than 10

- For the function of $P_{2}(\mathrm{E})$,

$\mathrm{T}_{1}$ and $\mathrm{T}_{2}$ must be equal.

\section{RESUlts AND Discussions}

Estimation of combustion performance of coal by improving it in aqueous environment has been proved by experimental studies of Cemil and Ece (2015). As a result of this study, a reduction in the $\mathrm{CO}_{2}$ value of the improved coal has been achieved by reducing the volatilization start temperature, which has resulted in an easier combustion of coal and a reduction of $\mathrm{CaO}$ and $\mathrm{Fe}_{2} \mathrm{O}_{3}$, which should be low for environmental combustion of the coal [15].

\section{REFERENCES}

[1] 8. Awards, COSPAR Information Bulletin 2004 (2004) 56-58.

[2] Swendsen RH, An introduction to statistical mechanics and thermodynamics, Oxford University Press, New York ; Oxford, 2012.

[3] Buonsante P, Franzosi R, Smerzi A, (2016) On the dispute between Boltzmann and Gibbs entropy, Ann Phys 375: 414-434.

[4] Tisza L, Quay PM, (1963) The statistical thermodynamics of equilibrium, Annals of Physics 25: 48-90.

[5] Rosen J, (2004) Encyclopedia of Physics Facts on File.

[6] Langer JS, (1967) Theory of the condensation point, Annals of Physics 41: 108-157.

[7] Turkevich LA, Doniach S, (1982) Critical behavior of the one-dimensional commensurateincommensurate transition, Ann Phys 139: 343-418.

[8] Anslyn EV, Dougherty DA, (2006) Modern Physical Organic Chemistry University Science.

[9] Pan Y, Yang Y-X, Chang N, Wang Z, Guan W, Yang J-Z, (2018) A new theoretical model - The ionic molar surface Gibbs free energy and its application, J Chem Thermodyn 116: 107-113.

[10] Wei J, Fan B-H, Pan Y, Xing N-N, Men S-Q, Tong J, Guan W, (2016) Vaporization enthalpy and the molar surface Gibbs free energy for ionic liquids [CnDmim][NTF2] $(\mathrm{n}=2,4), \mathrm{J}$ Chem Thermodyn 101: 278-284.

[11] Arzpeyma G, Gheribi AE, Medraj M, (2013) On the prediction of Gibbs free energy of mixing of binary liquid alloys, J Chem Thermodyn 57: 82-91.

[12] ter Horst HJ, (1987) Fundamental functions in equilibrium thermodynamics, Ann Phys 176: 217.

[13] Fisher ME, Felderhof BU, (1970) Phase transitions in one-dimensional cluster-interaction fluids Critical behavior, Ann Phys 58: 217-267.

[14] Stoitsov MV, Petkov IZ, (1988) Density functional theory at finite temperatures, Ann Phys 184: 121-147.

[15] Cemil K, Ece P, (2017) Biological Improvement of Coal: Formica rufa Enzymes over Mining Fluid and Rumen Liquid, Adv Energy Pow 5: 58-62.

Citation: Cemil Koyunoğlu, (2019) " Showing Relationship in Between a Same Reaction in both Liquid and Gas Phase by Monatomic Oxidation Hypothesis", International Journal of Research Studies in Microbiology and Biotechnology (IJRSMB), vol. 5, no. 3, pp. 3-6, 2019. Available: DOI: http://dx.doi.org/ 10.20431/24549428.0503002

Copyright: () 2019 Authors. This is an open-access article distributed under the terms of the Creative Commons Attribution License, which permits unrestricted use, distribution, and reproduction in any medium, provided the original author and source are credited. 